Revue québécoise de linguistique

--> Voir l'erratum concernant cet article

\title{
Analyse phonologique des emprunts en dida de Niakassé
}

\section{Monik Charette}

Volume 14, numéro 1, 1984

Grammaires en contact

URI : https://id.erudit.org/iderudit/602529ar

DOI : https://doi.org/10.7202/602529ar

Aller au sommaire du numéro

Éditeur(s)

Université du Québec à Montréal

ISSN

0710-0167 (imprimé)

1705-4591 (numérique)

Découvrir la revue

Citer cet article

Charette, M. (1984). Analyse phonologique des emprunts en dida de Niakassé. Revue québécoise de linguistique, 14(1), 87-111. https://doi.org/10.7202/602529ar d'utilisation que vous pouvez consulter en ligne. 


\title{
ANALYSE PHONOLOGIQUE DES EMPRUNTS EN DIDA DE NIAKASSÉ*
}

\author{
Monik Charette
}

\section{Introduction}

Nous nous proposons de faire une analyse phonologique des mots empruntés au français et à l'anglais ${ }^{1}$ dans une langue de la Côte-d'Ivoire : le dida de Niakassé.

Le dida, comme beaucoup d'autres langues de la même famille, a emprunté plusieurs mots au français et à l'anglais. Le nom est toutefois la seule catégorie lexicale empruntée. On ne retrouve aucun verbe, aucun adjectif, etc. ayant comme langue source l'anglais ou le français.

Le dida a une structure syllabique différente de celle du français et de l'anglais. Nous proposons dans un premier temps d'analyser ce qu'il advient de la structure syllabique des mots empruntés. Nous verrons que lorsque les emprunts possèdent une structure syllabique qui n'est pas permise dans la langue emprunteuse, ils subissent une modification au sein de leur structure, c'est-à-dire qu'ils deviennent conformes à la structure du dida.

Un second point qui retiendra notre attention concerne les tons. Le dida est une langue à tons; il nous paraît de ce fait intéressant d'observer les

* Cette recherche en linguistique africaniste est subventionnée par le Conseil de recherches en sciences humaines du Canada (\#410-81-0503). Je voudrais adresser mes remerciements à Jonathan Kaye, Jean Lowenstamm, Claire Lefebvre et Raj Singh pour les encouragements, conseils et remarques qu'ils m'ont apportés. Je remercie aussi M. Sako N'Guessan et les villageois du village de Niakassé pour les renseignements qu'ils m'ont fournis et la patience qu'ils m'ont témoignée. Je voudrais souligner qu'une version préliminaire de cet article a été présentée dans le cadre d'un séminaire de «Langues en contact» dirigé par Claire Lefebvre et Raj Singh, séminaire que j'ai suivi à l'Université du Québec à Montréal à la session d'hiver 1983.

1. Il est possible et même probable que certains emprunts du dida ne viennent qu'indirectement de la langue source. Ces mots auraient été empruntés directement à une autre langue africaine, par exemple au baoulé. Ceci vaut surtout pour les emprunts d'origine anglaise. 
patrons tonals des mots empruntés à des langues qui n'ont pas de tons. Les patrons tonals sont-ils prévisibles, et ce selon des principes phonologiques précis? L'accent des noms empruntés au français et à l'anglais est-il exprimé par les tons ou est-il simplement non considéré?

Une analyse approfondie des patrons tonals des emprunts nous dévoilera que les mots empruntés ont un patron tonal tout à fait prévisible et caractéristique des patrons acceptables du dida. De plus nous verrons que l'accent du français et de l'anglais est exprimé par un patron tonal précis.

Ce sont des problèmes pour lesquels nous tenterons de fournir une analyse phonologique, analyse qui se situe dans le cadre de la théorie métrique et tridimensionnelle.

2. Le dida de Niakassé

Le dida est un complexe de variétés de langues africaines parlées en Côte-d'Ivoire et qui appartiennent à la famille linguistique kru. Ce complexe ou groupe de parlers dida se divise en deux groupes linguistiques : le dida $\mathrm{k}^{\mathrm{W}}$ et le dida $\mathrm{F}$ (Kaye 1982). La variété sur laquelle portera mon étude fait partie du groupe dida $\mathrm{k}^{\mathbf{w}}$. Elle est appelée dida de Niakassé ou de Yocouboué parce qu'elle est parlée principalement dans les deux villages qui portent ces noms.

\subsection{Le système vocalique}

Le système vocalique du dida de Niakassé comprend dix voyelles qui se divisent en deux groupes ATR (advanced tongue root) : les voyelles rétractées (-ATR) et les voyelles avancées (+ATR). En fait, les éléments syllabiques sont au nombre de cinq, dont cinq voyelles rétractées qui ont chacune une correspondante avancée. Voici en (1) le tableau du système vocalique.

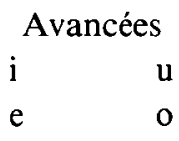

$(\Lambda)$

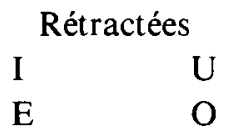

a

Nous remarquons que chacune des voyelles rétractées a sa correspondante avancée ${ }^{2}$.

2. Chacune des voyelles rétractées possède une correspondante avancée bien que le statut de $\mid \Lambda /$, correspondante avancée de /a/, soit controversé. Cette voyelle basse avancée n'est phonétiquement pas présente en dida de Niakassé, mais certaines évidences qui ne seront pas discutées ici, semblent laisser croire qu'elle le serait phonologiquement. Cette question n'a toutefois pas d'implications dans la présente étude. 
Voici en (2) un tableau du système vocalique sous forme de traits.

$\begin{array}{lllllllllll}\text { Haut } & + & + & - & - & - & + & + & - & - & - \\ \text { Bas } & - & - & - & - & + & - & - & - & - & + \\ \text { Arrière } & - & + & - & + & + & - & + & - & + & + \\ \text { Arrondi } & - & + & - & + & - & - & + & - & + & - \\ \text { ATR } & + & + & + & + & + & - & - & - & - & -\end{array}$

$\mathrm{Au}$ sein d'un mot, les éléments syllabiques s'accordent généralement pour la valeur du trait ATR. Les mots sont ainsi composés soit de voyelles avancées, soit de voyelles rétractées, mais il y aura rarement un conflit dans la valeur de ce trait.

(3)

\begin{tabular}{|c|c|c|c|}
\hline Avanc & & Rétracte & \\
\hline būdō & 'laver' & pàlú & 'maison' \\
\hline būtì & 'piler' & gōlÚ & 'pirogue' \\
\hline bès $\overline{1}$ & 'bananes' & $\mathrm{t} \overline{\mathrm{E}} \mathrm{l} \overline{\mathrm{I}}$ & 'tailler' \\
\hline līblē & 'oiseau', & kÓlā & "tortue", \\
\hline
\end{tabular}

Cela s'explique par la présence d'une harmonie dominante du trait ATR en dida (Vergnaud et Halle 1981, Kaye 1983a). De façon très générale, l'harmonie dominante est la présence, sur la couche autosegmentale, d'un trait harmonique flottant, ici + ATR, qui, lorsqu'il est présent, se propage sur tous les éléments syllabiques dans son domaine qui est le mot. Il est donc postulé qu'au niveau segmental, toutes les voyelles auront la valeur non marquée rétractée et qu'elles seront réalisées avancées si le trait harmonique + ATR est présent. Notons que la valeur du trait au plan harmonique prime sur celle au plan segmental.

\subsection{Le système consonantique}

Le système consonantique du dida de Niakassé comprend vingt-cinq consonnes, représentées dans le tableau suivant :

(4)

$\begin{array}{lcccccc}\text { occlusives : } & \mathrm{p} & \mathrm{t} & \mathrm{c} & \mathrm{k} & \mathrm{k} & \mathrm{kp} \\ & \mathrm{b} & \mathrm{d} & \mathrm{j} & \mathrm{g} & \mathrm{gw}^{\mathrm{w}} & \mathrm{gb} \\ \text { fricatives : } & \mathrm{f} & \mathrm{s} & & & & \\ & \mathrm{v} & \mathrm{z} & & & & \\ \text { nasales : } & \mathrm{m} & \mathrm{n} & \mathrm{n} & \mathrm{\eta} & & \\ \text { approximantes : } & \mathrm{b} & \mathrm{l} & \mathrm{y} & \gamma & \mathrm{w} & \end{array}$




\subsection{Les tons}

Les langues de la famille linguistique kru sont des langues à tons, qui sont phonétiquement au nombre de trois en dida de Niakassé3 ${ }^{3}$.

(5)

$$
\begin{aligned}
& \text { Haut : V́ } \\
& \text { Moyen : } \overline{\mathrm{V}} \\
& \text { Bas : ̀̀ }
\end{aligned}
$$

Les tons sont portés par les éléments syllabiques. Toutes les voyelles portent au moins un ton. 11 arrive que plus d'un ton soit attaché à une même voyelle, ce qui produit une séquence tonale appelée modulation tonale.

Les nasales syllabiques sont aussi porteuses de tons, et ce au même titre que les voyelles.

(6) प̣̣ lì sáká 'je mange du riz'

$$
\begin{array}{ll}
\text { ̣̀ } 1 \overline{1} \text { sáká } & \text { 'tu manges du riz' } \\
\bar{O} \mathrm{li}^{-} \text {sáká } & \text { 'il mange du riz' }
\end{array}
$$

3. La structure syllabique.

La présente section portera sur la structure syllabique du dida de Niakassé, du français et de l'anglais. Soulignons que l'analyse et les faits sont basés sur les travaux de Kaye et Lowenstamm (1984) portant sur leur théorie de la syllabe.

Une syllabe comprend minimalement deux constituants; une attaque et une rime. Le constituant de gauche est l'attaque, et celui de droite la rime.

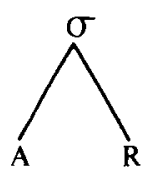

$\sigma$ représente une syllabe

A représente l'attaque

$R$ représente la rime

L'attaque et la rime peuvent se diviser en branches, mais cela dépend de la structure syllabique de la langue, soit des syllabes possibles dans une langue donnée. Si la rime entraîne un branchement, le constituant de gauche est le noyau et celui de droite la queue.

3. Phonétiquement parlant. Je laisse de côté la question d'un quatrième ton sous jacent. 
(8)

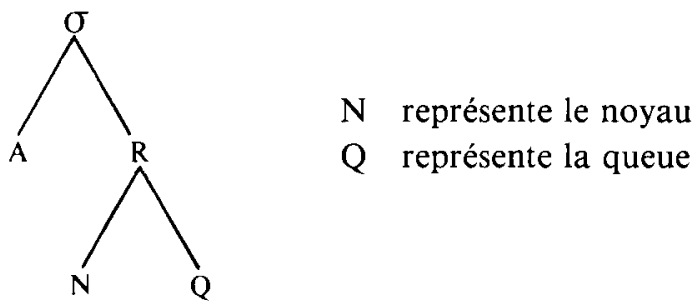

Chacun des constituants syllabiques est relié à un point terminal présent sur la couche du squelette ou épine. À ces points sont attachés les segments. Tout segment doit être relié à au moins un point du squelette et tout point doit être dominé par un constituant.

(9)

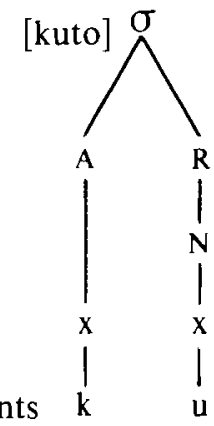

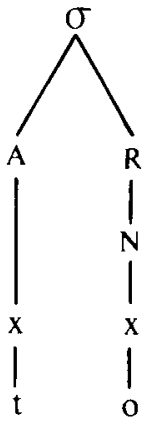

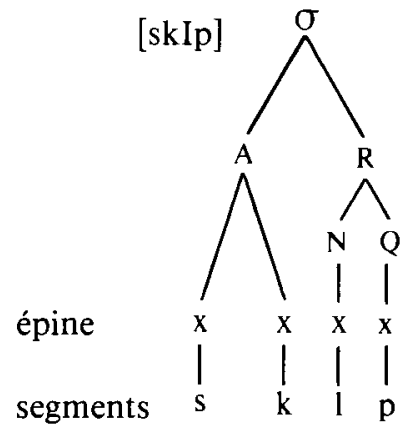

\subsection{La structure syllabique du dida de Niakassé}

La structure syllabique du dida est différente de celle du français et de l'anglais. En dida on n'y retrouve ni attaques, ni rimes branchantes. Le fait que la rime ne puisse pas entraîner un branchement signifie que la langue ne possède que des syllabes ouvertes, soit des syllabes où la rime n'est constituée que d'un noyau et non d'une queue.

(10) pi 'préparer'
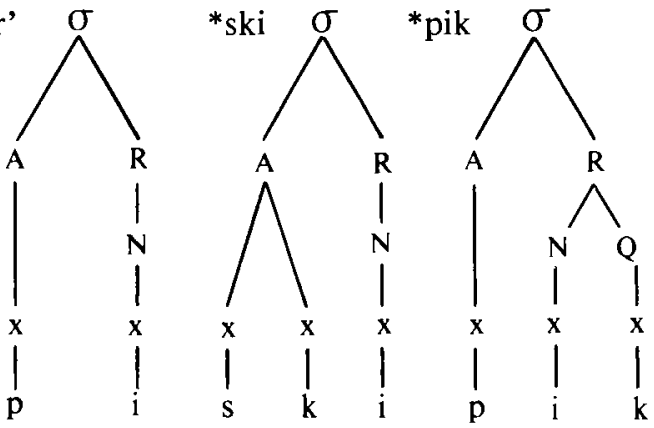
L'éventail des syllabes du dida est le suivant :

\begin{tabular}{|c|c|}
\hline (11) $\mathrm{CV}$ : & $\begin{array}{l}\text { lì } \\
\text { sáká }\end{array}$ \\
\hline V: & $\begin{array}{l}\text { à } \\
\text { ̀̀ }\end{array}$ \\
\hline
\end{tabular}

Les représentations de la structure syllabique des syllabes sont les suivantes :

(12) a. CV

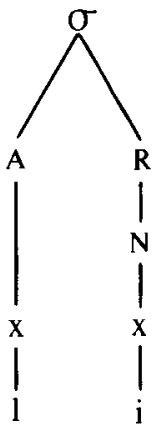

b. V

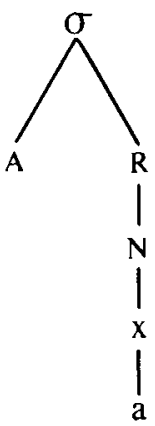

En (12 a) la syllabe comprend un branchement binaire qui est formé des constituants attaque et rime. Ni l'attaque ni la rime ne branchent, ce qui signifie que la rime ne domine que le noyau et non une queue. En (12 b) le constituant attaque est présent, mais il ne domine aucun point du squelette. C'est la configuration non marquée d'une attaque vide. Un constituant attaque qui ne domine aucun point du squelette ne domine aucun segment de la couche segmentale. L'attaque est donc vide et seul le noyau domine un point de l'épine auquel est attaché un segment.

Certains mots peuvent suggérer que la structure syllabique du dida comprend aussi des syllabes à attaque branchante.

$$
\begin{array}{ll}
\text { Glá } & \text { 'frapper/tuer' } \\
\text { srá } & \text { 'construire' } \\
\text { gblà } & \text { 'grimper' } \\
\text { blū } & \text { 'un' }
\end{array}
$$

Le fait qu'en dida l'attaque semble pouvoir brancher alors que la rime ne le peut pas, va à l'encontre de la théorie de Kaye et Lowenstamm (1981), à savoir que dans une langue, l'attaque ne peut être plus marquée que la rime. 
Les deux constituants peuvent avoir un indice de marque égal, mais l'attaque ne peut être plus marquée. Si en dida l'attaque branche et la rime ne branche pas, l'attaque aura donc un indice de marque plus élevé que celui de la rime.

Face à ce problème, Kaye (1983b) a montré que les syllabes du type de celles présentées en (13) ne sont non pas constituées d'une attaque branchante, mais plutôt d'une diphtongue légère ${ }^{4}$. Ainsi, la structure syllabique d'un verbe tel que blá 'frapper' est la suivante, où lá constitue une diphtongue légère, soit deux segments reliés à un seul point de l'épine.

(14)
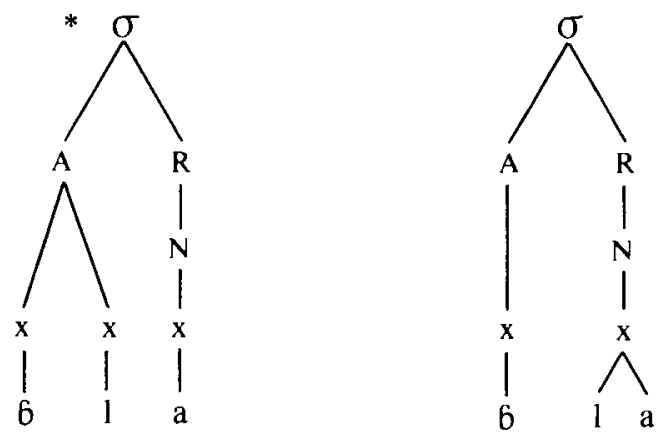

Soulignons ici que le deuxième élément de la suite consonantique est toujours une consonne liquide et qu'elle peut être précédée par n'importe quelle consonne de la langue, ce qui serait impossible si elle constituait une attaque branchante. La suite liquide-voyelle aura la même représentation que celle des diphtongues légères qui existent d'une manière indépendante dans la langue.

(15) plā 'acheter'
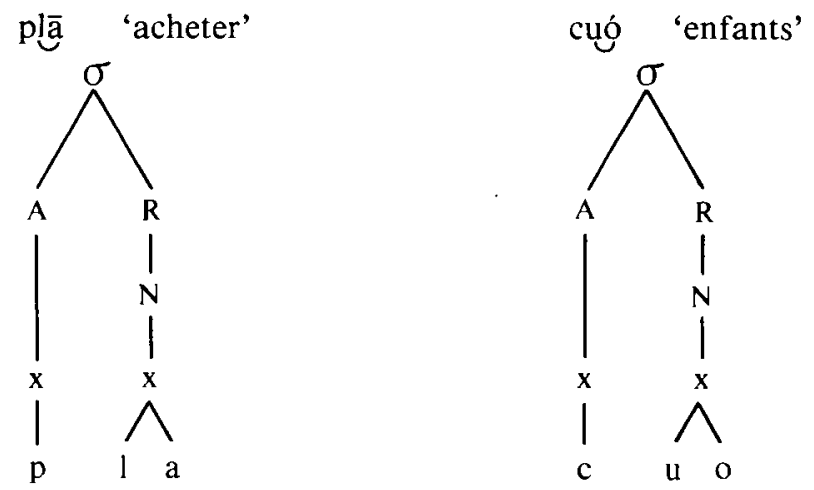

4. Voir Kaye (1983b.) pour l'argumentation. 


\subsection{La structure syllabique du français et de l'anglais}

Une analyse détaillée de la structure syllabique du français et de l'anglais n'est pas dans ce présent article justifiée. Nous n'en donnerons que les grandes lignes.

D'une manière différente du dida, le français et l'anglais ont un éventail de syllabes que l'on peut qualifier de «riches» ou de «complexes». Ces deux langues ont, en plus de syllabes ouvertes, des syllabes fermées, ce qui signifie que la rime peut brancher en un noyau et une queue.

(16) [kat]

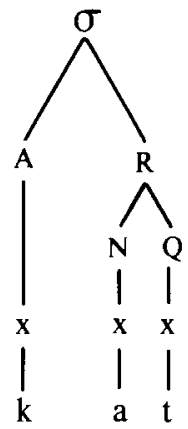

De plus, trois constituants de la syllabe, soit l'attaque, la rime et le noyau $\Lambda$ peuvent brancher, c'est-à-dire qu'ils peuvent dominer plus d'un point de l'épine 5 .

(17) attaque branchante rime branchante
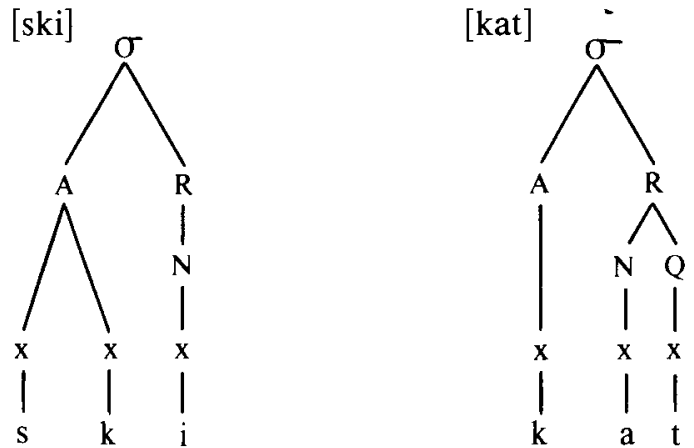

5. Je laisse de côté la question de la queue qui branche. Vu la présence d'un appendice final, il n'est pas évident qu'en français et en anglais la queue puisse brancher. Voir Charette (1983).

Dans cet article, suivant la théorie la moins controversée, je considérerai les éléments consonantiques finaux comme étant dominés par le noeud queue et non par un appendice final. 


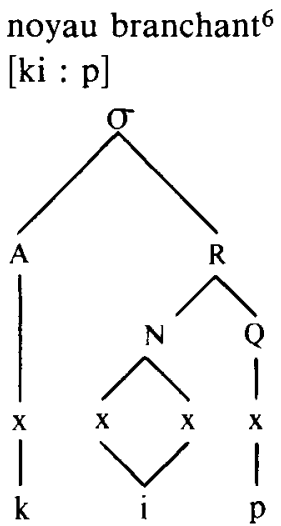

Nous remarquons que le français et l'anglais ont un éventail de syllabes plus considérable que celui du dida. Les constituants attaque, rime et noyau peuvent brancher, alors que cela est impossible dans la langue africaine. Chez cette dernière aucun constituant n'a la possibilité d'avoir un branchement.

La structure syllabique de la langue emprunteuse ainsi que celle des langues prêteuses ayant été présentées, nous aborderons l'analyse de la structure syllabique des noms empruntés au français et à l'anglais en dida.

\subsection{La structure syllabique des emprunts}

Le français et l'anglais ont une structure syllabique différente de celle du dida : leurs indices de marque de l'attaque et de la rime sont plus marqués que ceux des deux constituants de la syllabe de la langue africaine.

Qu'advient-il alors de la structure syllabique des mots empruntés, lorsque la structure de ces emprunts n'est pas possible dans la langue emprunteuse?

Notre hypothèse est que la langue emprunteuse (le dida), modifiera la structure syllabique du mot emprunté si sa structure n'est pas possible dans cette langue qui emprunte. La structure de l'emprunt sera donc modifiée pour devenir une structure possible et permise en dida. Ainsi, les syllabes qui constituent le mot emprunté et qui ne sont pas des syllabes possibles en dida seront modifiées pour devenir conformes à la structure syllabique de la langue africaine.

6. Le noyau branchant est possible en anglais et en québécois, mais non en français standard qui n'a ni voyelles longues, ni diphtongues lourdes. 
Observons un premier groupe de données :

\begin{tabular}{|c|c|}
\hline \multicolumn{2}{|l|}{ français / anglais } \\
\hline$[\mathrm{kafe}] \quad] \mathrm{kO}: \mathrm{fi}]$ & {$[$ kàfī $]$} \\
\hline [mamã] & [màmă \\
\hline [balō] & [bàlô] \\
\hline [kakao] & [kàkàớ] \\
\hline [pakE] & [pàk $\overline{\mathrm{E}}]$ \\
\hline [matəla] & tì̀lāa \\
\hline
\end{tabular}

Nous remarquons que lorsque la structure syllabique des mots empruntés est conforme à la structure syllabique du dida (des syllabes ouvertes sans constituant branchant), aucun changement ne se produit. Le mot est emprunté tel quel, sans que sa structure ne soit modifiée.

Considérons maintenant des emprunts qui contiennent une syllabe fermée finale, syllabe qui n'est pas permise en dida.

\subsubsection{Mots empruntés qui ont une syllabe fermée}

Nous savons que le dida n'a pas de syllabes fermées, c'est-à-dire des syllabes dont la rime branche en un noyau et une queue. Les seules syllabes du dida sont dites ouvertes, soit de type CV et V. Qu'advient-il alors de la structure syllabique des mots empruntés qui sont constitués d'une syllabe fermée?

\begin{tabular}{|c|c|}
\hline \multicolumn{2}{|l|}{ s/anglais } \\
\hline [tOmat] / & [tÙmát İ ] \\
\hline [mOnik] & [mòníkī] \\
\hline$[$ sigarEt] / [sIg $\partial \mathrm{rEt}]$ & [sìgàrÉt İ ] \\
\hline [bik] / [blk] & [bík̄i ] \\
\hline / [baeg] & [bág $\bar{I}$ ] \\
\hline$[\mathrm{arab}] / /$ [arab] & [àrábÛ] \\
\hline [kylOt] & 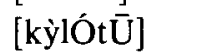 \\
\hline$[\mathrm{fO}: \mathrm{k}]^{7}$ & [fók İ ] \\
\hline [sak] / [saek] & [sák I ] \\
\hline [mObilEt] & [mÒbilét Ī ] \\
\hline [јّob] / [јّab] & [zúbū] \\
\hline
\end{tabular}

7. J'ai transcrit le mot «fork" sans le $/ \mathrm{r} /$, car dans les dialectes anglais parlés en Afrique, le $/ \mathrm{r} /$ n'est habituellement pas présent phonétiquement. Il n'y aurait donc pas eu de perte du $/ \mathrm{r} /$ en dida, mais absence de ce phonème dans le nom de la langue prêteuse. 
Nous remarquons que le dida n'emprunte pas sans la modifier une syllabe fermée. La stratégie employée afin de briser la syllabe fermée est de créer une voyelle épenthétique pour ainsi former deux syllabes ouvertes avec la syllabe fermée de l'emprunt.

En d'autres mots, la voyelle épenthétique permet la création d'une nouvelle syllabe ouverte avant comme constituant attaque la consonne qui se trouve dans la queue de la syllabe fermée de l'emprunt. Il y a donc resyllabification qui déplace sous une attaque suivante une consonne se trouvant sous un noud queue.

Observons en (20) la représentation syllabique des mots empruntés.

(20) [bik]

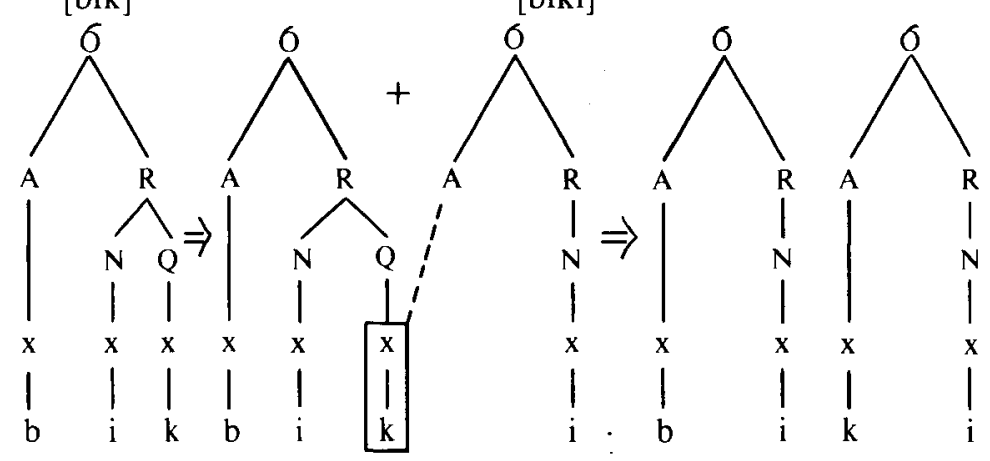

[tOmat]

[tÒmát $\overline{\mathrm{I}}$ ]

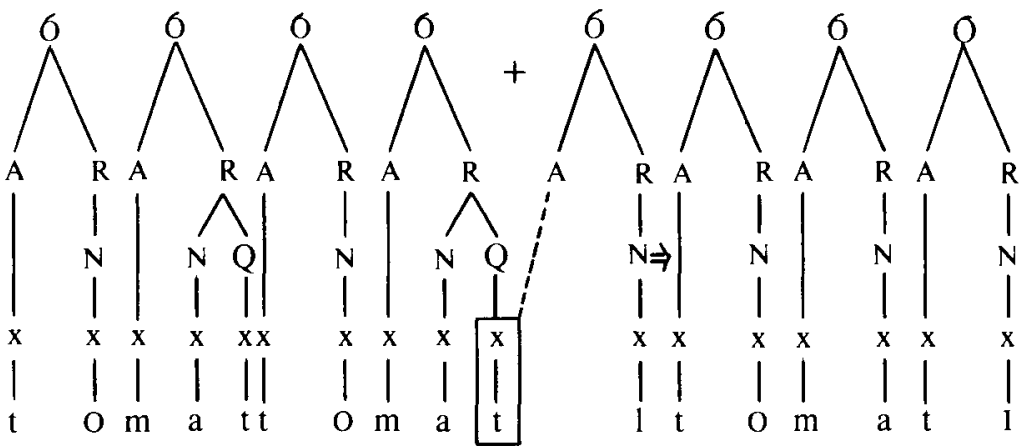

3.3.2 Emprunts à attaque branchante

(21)

français

[triko]

[plym]

[aprãti] dida

[trik $\dot{\mathrm{O}}$ ]

[plýmū]

[àplằtí ] 


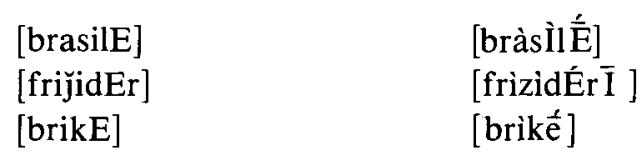

Nous observons que lorsque l'emprunt a une attaque branchante, la suite des deux consonnes est conservée en dida. Aucune voyelle épenthétique ne vient briser la suite consonantique. Si le dida accepte des attaques et non des rimes branchantes, cela va à l'encontre de la théorie du marquage syllabique, comme nous l'avons déjà mentionné. Ici, l'indice de marque de l'attaque serait plus élevé que celui de la rime.

Cependant, observons les données de plus près. Nous remarquons que chacune des attaques branchantes des noms empruntés est constituée d'une consonne suivie d'une liquide. Or, il existe des mots ayant cette configuration en dida. Voici les données de (13) représentée en (22).

(22)

$\begin{array}{ll}\text { blá } & \text { 'frapper' } \\ \text { slá } & \text { 'construire' } \\ \text { gblá } & \text { 'grimper' } \\ \text { blū } & \text { 'un' }\end{array}$

Nous avons déjà établi que la représentation syllabique de tels radicaux est une attaque non branchante suivie d'un noyau qui domine une diphtongue légère.

slá 'construire'

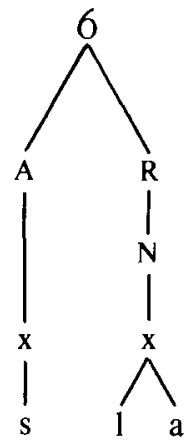

Cette structure syllabique existe déjà dans la phonologie du dida; il n'y a rien d'étonnant à la retrouver dans les emprunts. L'attaque branchante consonne-liquide du nom emprunté ne sera non pas simplifiée par 
l'insertion d'une voyelle épenthétique (*birikE), mais plutôt interprétée comme une diphtongue légère ${ }^{8}$.

(24)

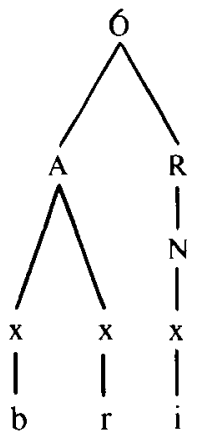

[brikE]

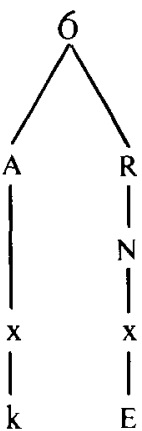

$\left[\right.$ brìkē ${ }^{9}$

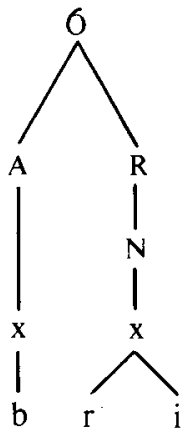

[plym]
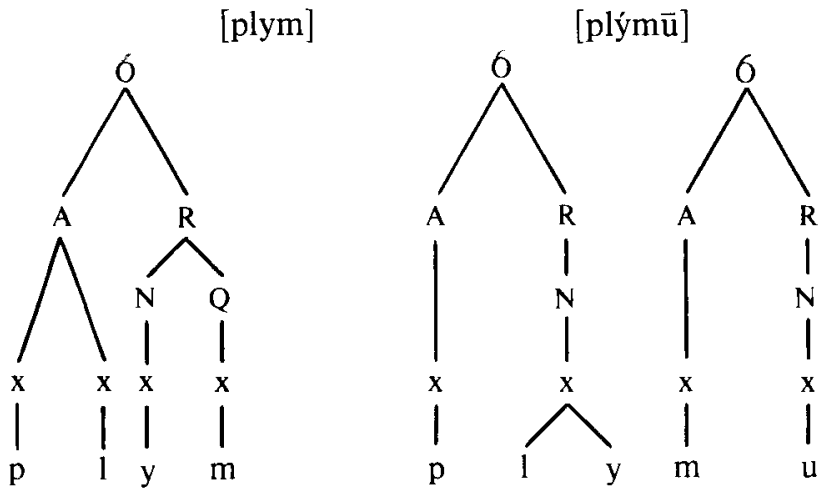

C'est pourquoi des noms empruntés qui sont formés d'une syllabe à attaque branchante consonne-liquide ne subissent pas, en dida, de modification au sein de la suite consonantique. Du fait qu'il existe déjà des diphtongues légères formées d'une consonne liquide et d'une voyelle, l'attaque branchante de l'emprunt sera tout simplement modifiée en attaque non branchante suivie d'une diphtongue légère sous le noyau.

8. En français la suite consonne-liquide constitue une attaque branchante et non une diphtongue légère. Voir Kaye (1983b).

9. Notons qu'il n'y a qu'une seule consonne liquide dans l'inventaire phonémique du dida. Cette liquide se réalise parfois [1], parfois [r] selon la consonne qui la précède. 


\subsubsection{Emprunts qui ont une suite consonantique}

Nous avons vu que le dida emprunte une suite consonantique constituée d'une consonne suivie d'une liquide. Observons s'il en va de même pour une suite consonantique constituée de deux consonnes.

\begin{tabular}{|c|c|}
\hline français / anglais & dida \\
\hline [skul] & [sùkúlī] \\
\hline [pnø] & [pìné] \\
\hline [baskEt] & [bàsìkÉt̄̄] \\
\hline [plastik] & [plàsitík̄i ] \\
\hline
\end{tabular}

Nous remarquons que les suites consonantiques formées de deux consonnes ne sont pas conservées en dida. Une telle suite consonantique n'étant pas possible dans la langue emprunteuse, une stratégie est employée afin de détruire cette suite de consonnes. Une voyelle épenthétique est introduite entre les deux consonnes, ce qui a pour effet de détruire les attaques branchantes ainsi que les syllabes fermées.
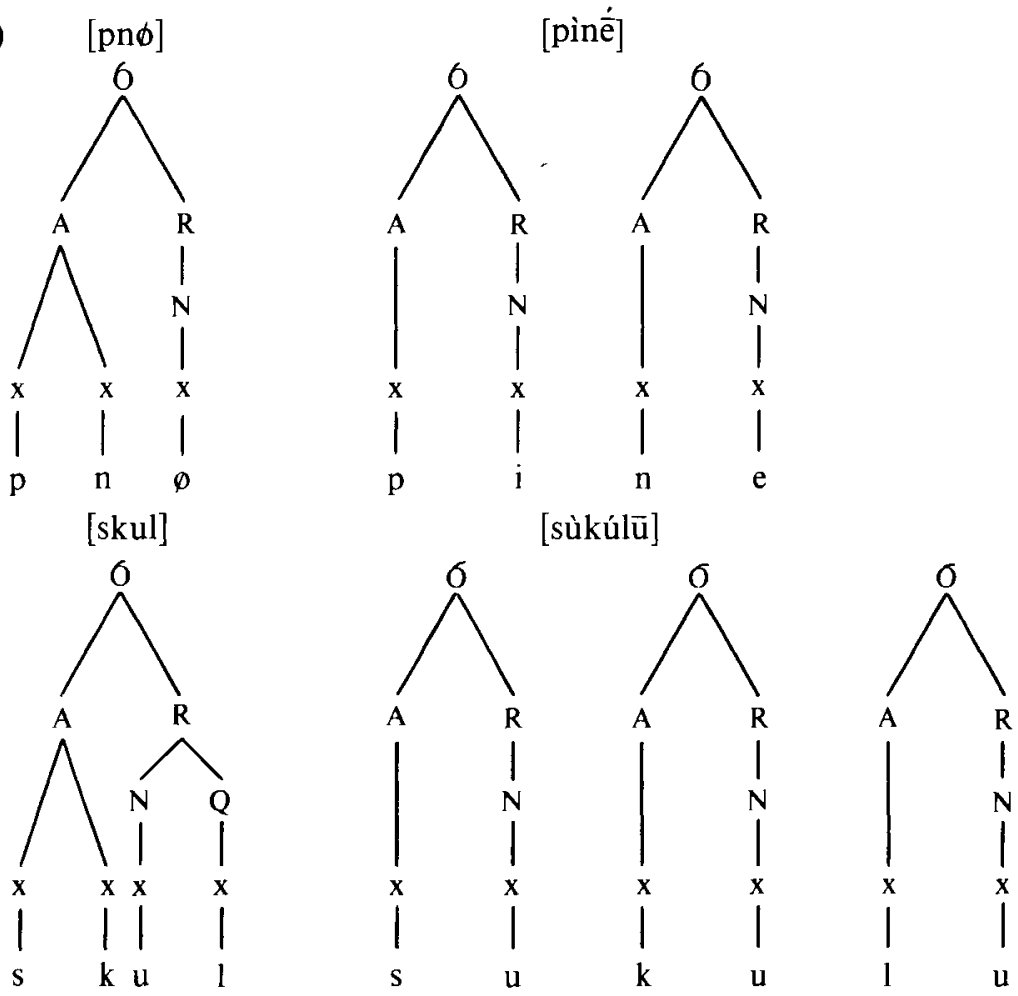
Une forme intéressante est celle présentée en (25). Le mot [plastik] est emprunté. La suite consonantique initiale /pl/ est conservée, étant constituée d'une consonne suivie d'une liquide. L'attaque branchante est simplement modifiée en attaque non branchante suivie d'une diphtongue sous le noyau. Par contre, la suite /st/ est détruite, car la consonne qui se trouve sous la queue ne peut être transportée sous une attaque suivante, ce dernier constituant étant déjà rempli. La voyelle épenthétique forme donc une syllabe ouverte ayant comme attaque le / s / se trouvant sous la queue de la syllabe de l'emprunt. Enfin, la dernière syllabe fermée est aussi modifiée en deux syllabes ouvertes. Voici en (27) la représentation syllabique de ce mot.

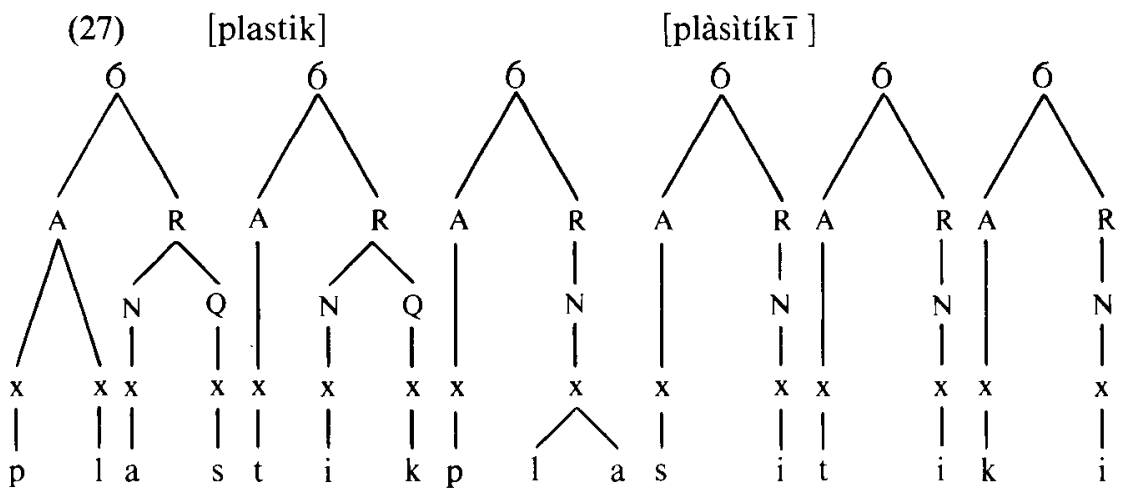

Avant de clore ce chapitre sur la structure syllabique des emprunts, nous aimerions préciser que les diphtongues légères du dida peuvent avoir comme segments reliés au noyau une voyelle haute ou une consonne liquide, suivie d'une voyelle. En ce qui concerne la consonne, seule une liquide peut occuper cette position sous le noyau. Toutefois, on peut penser qu'une nasale puisse en surface occuper cette position.

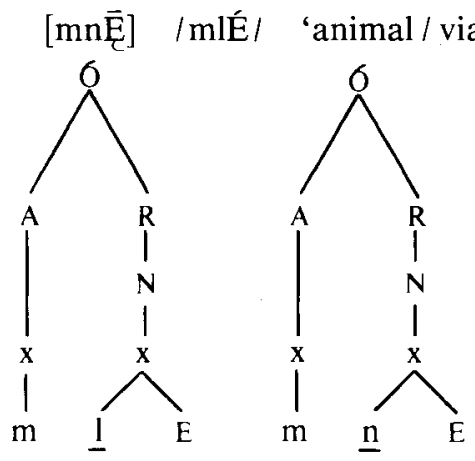


Cependant une consonne nasale ne peut occuper cette position sous le noyau. Le segment en question est une consonne liquide qui a subi une nasalisation par le biais de la consonne qui la précède. La présence de [n] plutôt que [1] ou [r] est prévisible. On ne retrouve [n] qu'après une consonne nasale, et jamais après une orale. De plus, les voyelles (éléments nucléaires) se nasalisent après une consonne nasale; nŪ 'boisson' [nU్]]. Puisque nous avons proposé que la liquide, dans les séquences CLV, est sous le noyau, on s'attend donc à ce qu'elle se nasalise après une consonne nasale. Or, cela est parfaitement vrai, comme le montrent des formes telles que [mnEৃ] $]$. Une diphtongue légère formée d'une réelle consonne nasale suivie d'une voyelle n'est pas une diphtongue possible en dida. Cela explique pourquoi la suite consonantique /pn/ du mot pneu, n'est pas considérée comme une diphtongue légère en dida, le $/ \mathrm{n} /$ étant une nasale et non une liquide nasalisée.

D'autre part, il serait intéressant d'essayer de prédire l'identité de la voyelle épenthétique. Prenons comme exemple le mot bik. À notre avis, il n'existe que deux solutions si on veut respecter la structure syllabique du dida. On peut laisser tomber la dernière consonne $\left({ }^{*} \mathrm{bi}\right)$, ou ajouter une rime (un noyau V). La première stratégie entraînera peut-être une perte d'information inacceptable. On prédit alors la deuxième stratégie, soit l'addition d'une voyelle pour remplir la rime. De façon générale, la voyelle choisie est I ( + ou - ATR selon la qualité ATR de la voyelle qui la précède ou la suit) lorsque précédée ou suivie d'une voyelle non arrondie (mObilEtI, biki, pine). La voyelle se manifestera $U$ ( + ou - ATR) si elle est précédée ou suivie d'une voyelle arrondie, ou lorsqu'elle est placée avant ou après une consonne labiale (zubu, sukulu).

4. Les patrons tonals des mots empruntés.

Le second point que nous tenterons d'analyser concerne les patrons tonals des noms empruntés.

Nous savons que le dida est une langue à tons qui sont phonétiquement au nombre de trois, tandis que le français et l'anglais sont des langues à accent. Nous tenterons donc d'analyser les patrons tonals réservés aux noms empruntés à des langues qui n'ont pas de tons. Ces patrons sont-ils prévisibles, et si oui, selon quels principes phonologiques? 
Considérons les formes suivantes :

\begin{tabular}{|c|c|c|}
\hline $\begin{array}{l}\text { (29) Monosyllabiques } \\
\text { [dé] }\end{array}$ & 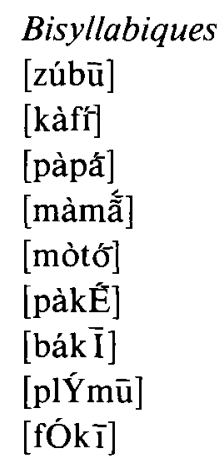 & $\begin{array}{l}\text { Disyllabiques } \\
\text { [tÙmát } \overline{\mathrm{I}} \text { ] } \\
\text { [mòník } \overline{1} \text { ] } \\
\text { [sìgàrÉt } \overline{\mathrm{I}} \text { ] } \\
\text { [bìsìklÉt } \overline{\mathrm{I}} \text { ] } \\
\text { [mòbilét } \overline{\mathrm{I}} \text { ] } \\
\text { [bàsikét } \overline{\mathrm{I}} \text { ] } \\
\text { [sùdúlū }] \\
\text { [plàsitík } \overline{1} \text { ] } \\
\text { [plàsitík } \overline{1} \text { ] }\end{array}$ \\
\hline
\end{tabular}

Tentons de mettre un peu d'ordre dans ces formes. Le seul emprunt monosyllabique que nous retrouvons a le patron tonal Haut-Moyen, soit une modulation tonale sur la voyelle. En fait, les tons sous-jacents attachés à la voyelle sont Haut-Bas, le dida ayant une règle phonétique d'élévation de tout ton Bas à Moyen en final de mot.

Les emprunts tout comme les mots natifs subissent cette règle d'élévation tonale.

$$
\text { Bas } \longrightarrow \text { Moyen I _ } \longrightarrow \text { \# }
$$

La représentation tonale du nom monosyllabique est la suivante :<smiles>[B][CH]</smiles>

Considérons maintenant les noms bisyllabiques. Nous remarquons qu'il y a deux groupes de patrons tonals. Le premier type de patron est un ton Bas associé à la première voyelle du nom, suivi d'une modulation HautBas, phonétiquement Haut-Moyen, sur le dernier élément syllabique.

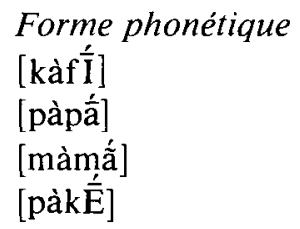

Forme sous-jacente
/ kàf
/pàpâ//
/màmẫ//
/pàkế/

Le second type de patron tonal est un ton Haut associé à la première voyelle et un ton Bas, phonétiquement Moyen, associé à la dernière. 
(33)

\begin{tabular}{|c|c|}
\hline Forme phonétique & Forme sous jacente \\
\hline [báḡ̄] & / bák $\overline{\mathrm{I}} /$ \\
\hline [zúbū] & /zúbù/ \\
\hline [bík̄̄] & /bíkì / \\
\hline [límō] & /límò/ \\
\hline [pílīi] & / pílì / \\
\hline [plýmū] & / plýmù / \\
\hline [fók $\overline{\mathbf{I}}]$ & / fókì/ \\
\hline
\end{tabular}

Devant ces faits, l'analyse que nous proposons est la suivante. L'accent $d u$ nom emprunté est interprété en dida par une suite de tons Haut-Bas. Ainsi, les syllabes qui portent l'accent dans les mots empruntés seront représentées en dida comme portant une suite de tons Haut-Bas sur cette syllabe.

(34) Règle d'interprétation de l'accent

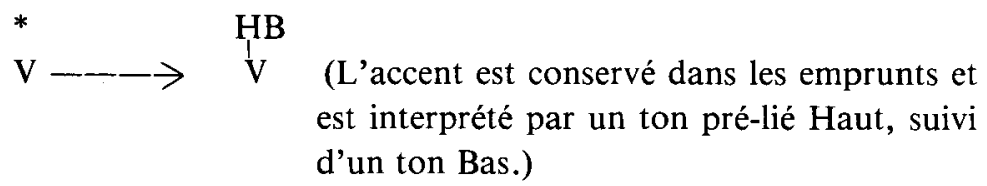

Reconsidérons les données de (32), où l'accent est placé sur la dernière syllabe du nom, accent que nous représentons par un astérisque.

$\begin{array}{ll}\begin{array}{l}\text { Langue source } \\ \text { [kafé] }\end{array} & \text { dida } \\ \text { [papàfî́] } & \text { [pàpá] } \\ \text { [mamá] } & \text { [màmà] } \\ \text { [pakË] } & \text { [pàkÊ] }\end{array}$

L'accent de l'emprunt étant placé sur la dernière syllabe, cette syllabe aura comme représentation tonale en dida une suite de tons Haut-Bas. Nous suggérons que cette suite tonale représente l'accent. Cela pourrait s'exprimer de la façon suivante :
Langue source
dida
C V
CQ V́ 
Le ton Haut est pré-lié à la voyelle qui porte l'accent (l'astérisque), ce qui explique la présence du ton Haut sur l'élément syllabique. Cependant, comment peut-on rendre compte de la modulation tonale Haut-Bas?

Nous savons que l'association des tons aux segments se fait de gauche à droite, dans une relation de un pour un. (Goldsmith 1976)

$$
\begin{array}{rrrr}
\mathrm{T} & \mathrm{T} & \mathrm{T} & \mathrm{T} \\
\mathrm{I} & \mathrm{I} & \mathrm{I} & \mathrm{I} \\
\mathrm{C} \mathrm{V} & \mathrm{C} \mathrm{V} & \mathrm{C} \mathrm{V} & \mathrm{C} \mathrm{V}
\end{array}
$$

En ce qui concerne les emprunts, le ton Haut s'associe à la voyelle de la syllabe accentuée, soit à la voyelle portant un astérisque. La modulation que nous observons s'explique par le fait que nous analysons l'accent comme une suite de tons Haut-Bas. Le ton Bas doit s'associer, suite à l'association du ton Haut, à la prochaine voyelle à la droite de celle qui est accentuée. Vu l'absence d'élément syllabique suivant la voyelle accentuée (ex. 35), le ton Bas a comme seul point d'attache possible cette même voyelle accentuée, ce qui résulte en une modulation tonale sur cet élément syllabique ${ }^{10}$.

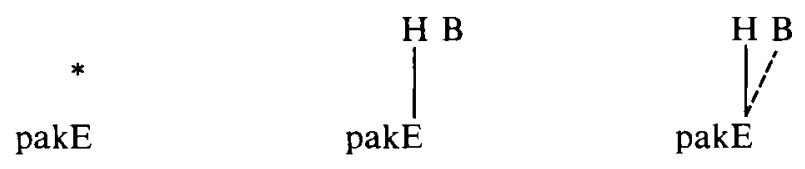

Le deuxième groupe de données, ci-dessous, a un patron tonal différent de celui des emprunts que nous avons observés plus haut.

\begin{tabular}{|c|c|}
\hline Langue source & dida \\
\hline [bäg] & [bák $\bar{I}]$ \\
\hline [jab] & [zúbū] \\
\hline [bik] & [bík̄i] \\
\hline$\left[I^{*} \mathrm{~m}\right]$ & [límō] \\
\hline [pill] & [pílī] \\
\hline [plÿm] & [plým̄̄] \\
\hline & fók $\mathrm{I}]$ \\
\hline
\end{tabular}

Il y a une différence entre les emprunts présentés en (35) et ceux présentés en (39). Les emprunts de (35) sont formés de deux syllabes

10. Je remets à plus loin l'explication du ton Bas associé aux premiers éléments syllabiques. 
ouvertes, alors que les noms empruntés de (39) sont composés d'une seule syllabe fermée.

Le dida n'apporte aucun changement à la structure des emprunts de (35), alors qu'il produit deux syllabes ouvertes avec la syllabe fermée des emprunts de (39), par le biais de l'épenthèse d'une voyelle.

Ainsi, chez les deux groupes d'emprunts, l'accent n'est pas placé sur la même syllabe. L'accent se trouve sur la dernière syllabe des noms bisyllabiques en (35) composés de deux syllabes ouvertes, alors qu'il se trouve sur la première syllabe, soit la seule syllabe des emprunts composés d'une seule syllabe fermée.

(40)

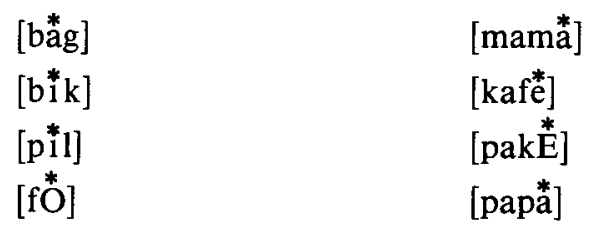

La position différente de l'accent explique la différence des patrons tonals.

Dans les emprunts monosyllabiques à syllabe fermée, l'accent se trouvant sur la première voyelle, c'est celle-ci qui porte le ton Haut. Le ton Bas peut s'associer à un élément syllabique à la droite de la voyelle accentuée, cet élément syllabique étant présent par le fait que la stratégie employée par le dida est de créer une voyelle épenthétique pour détruire la syllabe fermée de l'emprunt.

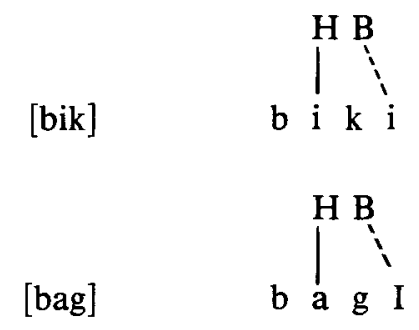

Dans les noms disyllabiques, les patrons tonals sont dérivables par notre analyse. 
(42)

[tOmat]

[skul]

[bisiklEt]<smiles>O=[13CH]I</smiles>

[tÒmát I]

[sùkúlū]

[bisiklÉt I] ]

Le ton Haut cherche la voyelle qui porte un astérisque (l'accent), et s'y attache. Le ton Bas qui le suit cherche à son tour un point d'attache et s'associe à toutes les voyelles qui suivent.

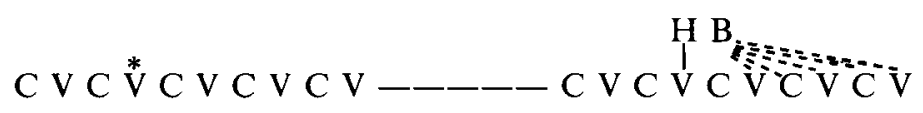

Considérons maintenant les tons associés aux éléments syllabiques qui précèdent la voyelle accentuée.

Nous remarquons que les voyelles qui précèdent la voyelle qui porte l'accent portent toutes un ton Bas. Nous avons deux hypothèses possibles pour rendre compte de ce phénomène.

La première hypothèse est que le patron tonal représentant l'accent de l'emprunt ne serait non pas la suite de tons Haut-Bas, mais plutôt la suite Bas-Haut-Bas. Ainsi, le ton Haut s'associerait à la voyelle accentuée et le ton Bas le précédant s'associerait à toutes les voyelles qui précèdent cette voyelle accentuée.

[bisiklEt]

[petrOlEt]

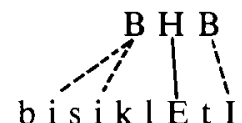

b is iklE t I

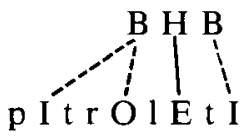

[bìsiklÉt I] ]

[pİtròlÉt $\bar{I}$ ] 
Mais si cette analyse est la bonne, comment expliquer que ce premier ton Bas ne s'associe pas dans les formes suivantes?
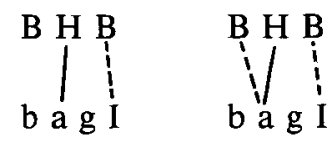

[bág $\bar{I}]$

[băg $\bar{I}]$

\begin{tabular}{|c|c|c|c|}
\hline fOk] & f O k I & f O k I & [fók̄̄] \\
\hline
\end{tabular}

S'il y avait un ton Bas précédant le ton Haut dans la représentation tonale de l'accent, rien n'empêcherait ce ton Bas de s'attacher à la première voyelle et de créer une modulation tonale Bas, Haut sur ce premier élément syllabique. Cette modulation tonale Bas, Haut est d'ailleurs permise en dida.

Nous devons donc rejeter cette première hypothèse. Nous en retiendrons une deuxième qui nous paraît être plus juste. L'accent du nom emprunté serait, comme nous l'avions jusqu'alors présumé, représenté par une suite tonale Haut-Bas. Le ton Haut est attaché, lié à la voyelle qui porte l'accent (un astérisque). Le ton Bas qui flotte cherche à son tour un point d'attache et s'associe à toute voyelle suivant celle qui porte l'accent ou le ton Haut. Si aucun élément ne suit la voyelle accentuée, le ton Bas s'attache à cette voyelle produisant une modulation tonale Haut, Bas.

Quant aux éléments syllabiques qui précèdent la voyelle accentuée, aucun ton ne peut leur être associé. (Le ton Haut ne peut se propager sur ces voyelles, ce ton étant un ton lié et non un ton flottant). Ils prennent alors la valeur d'un ton non marqué, qui est pour les radicaux nominaux en dida de Niakassé un ton Bas. Ainsi, toutes les voyelles précédant la voyelle accentuée, et ce peu importe leur nombre, porteront phonétiquement un ton Bas, soit la valeur non marquée.

Il reste toutefois une donnée qui soulève un problème intéressant. Le dida a emprunté un prénom de la bible : Abel. Le patron tonal associé à ce nom ne semble pas être dérivable par notre analyse. Observons la donnée :

$$
[\mathrm{abEl}] \longrightarrow \text { - } \longrightarrow \text { [F́bÈl̄̄ }]
$$

L'accent du nom en français se trouvant sur la dernière syllabe, nous nous attendrions au patron tonal suivant : 
(46)
[abEl]
[ÈbÉl̄̄]
E b E I I

Le patron tonal attendu n'est pas celui qui est réalisé. Supposons que le prénom n'ait pas été emprunté au français, mais à l'anglais. Si tel est le cas, l'accent de l'emprunt n'est non pas sur la dernière syllabe comme en français, mais placé sur la première syllabe.

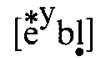

Si l'anglais est la langue source de l'emprunt, le patron tonal résultant est bien celui qui serait dérivé par notre analyse ${ }^{11}$.

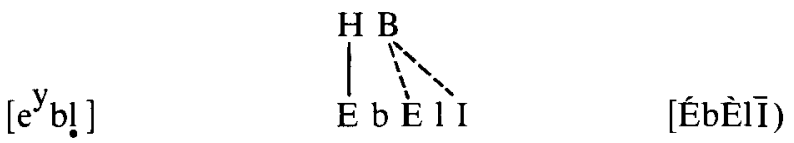

En outre, cette analyse tonale est confirmée par la prononciation des voyelles. Si le prénom avait été emprunté au français, le /a/ initial n'aurait eu aucune raison d'être modifié en [E], puisque cette voyelle /a/a toujours été conservée dans d'autres emprunts.

En résumé, notre analyse tonale des emprunts nous permet d'expliquer la réalisation de l'accent par les tons et aussi de rendre compte des faits historiques, à savoir à quelle langue un mot a été emprunté.

Quelle est par exemple la langue source de l'emprunt [plàsitíkī]? L'anglais ou le français? Appliquons notre analyse qui devrait répondre à cette question.

(49)
anglais : [plastIk]
[plásìtikī]

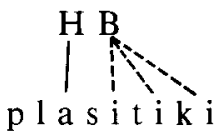

11. Je voudrais souligner qu'une autre solution pour le patron tonal des emprunts m'a été suggérée. Il s'avère toutefois que je ne la retiens pas pour les raisons suivantes. La solution est que seul un ton Haut serait associé à la voyelle accentuée (non la suite Haut-Bas). Toutes les voyelles non accentuées des mots empruntés prendraient la valeur du ton non marqué, le ton bas. À cette interprétation de l'accent s'ajouterait une règle insérant un ton Bas devant \#. Cette solution doit être rejetée à cause des mots natifs tels que : II 'chansons', IU 'chanson du village'...) qui ne se terminent pas par un ton Bas devant \#. 
français : [plastik] plas i ti k

[plàsìtík̄] ]

\section{Conclusion.}

Nous avons vu par le biais d'une analyse phonologique des mots empruntés en dida au français et à l'anglais, que tout emprunt est intégré dans la langue emprunteuse et ce en accord avec la phonologie de cette langue qui emprunte.

La langue qui emprunte ne modifiera pas sa structure, son système phonologique, afin d'intégrer des mots d'origine étrangère. Au contraire, ce sont les emprunts qui seront modifiés afin de devenir conformes à la structure et à la phonologie de la langue emprunteuse.

Il n'est jamais nécessaire d'utiliser des contraintes indépendantes et autonomes pour intégrer les mots étrangers. Tout découle au contraire de la phonologie de la langue emprunteuse.

D'autres phénomènes, que nous avons dû passer sous silence dans cette étude, devraient être considérés dans une analyse ultérieure. Par exemple, tout ce qui touche aux segments et à l'harmonie. Les segments des emprunts sont-ils toujours conservés? S'ils ne sont pas présents dans l'inventaire phonémique du dida, comment sont-ils réalisés? Le système harmonique est-il conservé dans les mots empruntés? S'il ne l'est pas, quelles violations sont permises et tolérées et quelles sont celles qui ne le sont pas? Quelle serait l'analyse des radicaux disharmoniques?

Plusieurs questions ont été posées et nous espérons avoir l'opportunité de les analyser dans le cadre des recherches ultérieures. 


\section{Références}

CHARETTE, M. (1983), "The Appendix in Parametric Phonology», à paraître dans Journal of African Linguistics, Proceedings du $15^{\mathrm{e}}$ colloque sur la linguistique africaniste.

GOLDSMITH, J. (1976), «Autosegmental Phonology», Indiana University Linguistics Club. Aussi publié en 1979 par Garland Press, New York.

HAllé, M. \& J.R. VergnaUd (1981), «Harmony Processes», dans W. Klein \& W. Levelt (éd.), Crossing the Boundaries in Linguistics, Reidel, 1-22.

KAYE, J.D. (1982), “Les dialectes dida» dans Projet sur les langues Kru, premier rapport de recherche, Université du Québec à Montréal.

KAYE, J.D. \& J. LOWENSTAMM (1984), «De la syllabicité» dans Forme sonore $d u$ langage - Structure des représentations en phonologie, Hermann, Paris, F. Dell, D. Hirst, J.R. Vergnaud, éditeurs.

KAYE, J.D. (1983a), "Harmony process in Vata" dans The Structure of Phonological Representation (Part two), Dordrecht, Foris Publications Holland, Harry Vander Hulst and Norval Smith, éditeurs.

KAYE, J.D. (1983b), «On the syllabe structure of certain West African languages», dans African linguistics, Essays in Memory of M.W.K. Semikenke, Amsterdam, J. Benjamins, Didier Goyvaerts, éditeurs. 